\title{
Electrical-transport studies on (La1-xPrx)2/3Ba1/3MnO3 compounds
}

\begin{abstract}
The magnetic and transport properties of (La1-xPrx)2/3Ba1/3MnO3 $(\mathrm{x}=0,0.1677,0.333$, $0.500,0.677,0.833,1.000)$ compounds, prepared by the solid state reaction have been investigated. The metal-insulator transition (Tp) was determined by using the standard fourpoint probe resistivity measurement between of 30 and $300 \mathrm{~K}$. With increasing Pr doping, Tp shifted to lower temperatures, which are greater than 300, 270, 250, 226, 202, 186 and $158 \mathrm{~K}$ for $\mathrm{x}=0,0.1677,0.333,0.500,0.677,0.833$ and 1.000 , respectively. By analyzing the data using several theoretical models, it was concluded that the metallic (ferromagnetic) part of the resistivity $(f)$, (below TP), fits well with the equation $f=f 0+f 2 T 2$. This indicates that $f 0$ is due to the grain/domain boundary effects. The second term $\sim 2 \mathrm{~T} 2$ appears to be attributed to electron-electron scattering. In the high temperature range ( $T>T P$ ) (the paramagnetic insulating regime) adiabatic small polaron models fit well in different temperature regions, thereby indicating that polaron hopping might be responsible for the conduction mechanism. The activation energy (Ea) also increases as the doping concentration increases.
\end{abstract}

Keyword: Metal-insulator transition temperature (Tp); Activation energy (Ea); Polaron 